\title{
On canonical quantization of the gauged WZW model with permutation branes
}

\author{
Gor Sarkissian * \\ Department of Theoretical Physics, Yerevan State University, \\ Alex Manooghian 1, Yerevan 0025, \\ Armenia
}

February 2011

\begin{abstract}
In this paper we perform canonical quantization of the product of the gauged WZW models on a strip with boundary conditions specified by permutation branes. We show that the phase space of the $N$-fold product of the gauged WZW model $G / H$ on a strip with boundary conditions given by permutation branes is symplectomorphic to the phase space of the double Chern-Simons theory on a sphere with $N$ holes times the time-line with $G$ and $H$ gauge fields both coupled to two Wilson lines. For the special case of the topological coset $G / G$ we arrive at the conclusion that the phase space of the $N$-fold product of the topological coset $G / G$ on a strip with boundary conditions given by permutation branes is symplectomorphic to the phase space of Chern-Simons theory on a Riemann surface of the genus $N-1$ times the time-line with four Wilson lines.
\end{abstract}

Keywords: conformal field theory, gauged WZW models, permutation branes, topological field theory, D-branes.

\footnotetext{
* Email address:

gor.sarkissian@ysu.am
} 


\section{Introduction}

In this paper we continue to investigate the problem of the canonical quantization of the WZW and gauged WZW models with defects and branes, started in the papers [19, 20]. In the paper [19] we addressed the problem of the canonical quantization of the WZW model with defects and permutation branes. In the paper [20] the canonical quantization of the gauged WZW model with defects has been performed. In the present paper we turn to the canonical quantization of the gauged WZW model $G / H$ with permutation branes. It was shown in the paper [19] that the phase space of the $N$-fold product of WZW models on a strip with boundary conditions given by permutation branes is symplectomorphic to the phase space of Chern-Simons theory on a sphere with $N$ holes times the time-line with two Wilson lines. Using the ansatz for the permutation branes on product of cosets suggested in [18], here we show that the phase space of the $N$-fold product of the gauged WZW models on a strip with boundary conditions given by permutation branes is symplectomorphic to the phase space of the double Chern-

Simons theory on a sphere with $N$ holes times the time-line with $G$ and $H$ gauge fields both coupled to two Wilson lines. For the case of the topological coset $G / G$ we get that the phase space of the $N$-fold product of the topological coset on a strip with boundary conditions given by permutation branes is symplectomorphic to the phase space of the Chern-Simons theory on a Riemann surface of the genus $N-1$ times the time-line with four Wilson lines.

\section{Bulk WZW model}

In this section we review the canonical quantization of the WZW model with compact, simple, connected and simply connected group $G$ on the cylinder $\Sigma=$ $R \times S^{1}=(t, x \bmod 2 \pi)[3,9,12$. The world-sheet action of the bulk WZW model is 21 ]

$$
\begin{aligned}
S^{\mathrm{WZW}}(g) & =\frac{k}{4 \pi} \int_{\Sigma} \operatorname{Tr}\left(g^{-1} \partial_{+} g\right)\left(g^{-1} \partial_{-} g\right) d x^{+} d x^{-}+\frac{k}{4 \pi} \int_{B} \frac{1}{3} \operatorname{tr}\left(g^{-1} d g\right)^{3} \\
& \equiv \frac{k}{4 \pi}\left[\int_{\Sigma} d x^{+} d x^{-} L^{\mathrm{kin}}+\int_{B} \omega^{\mathrm{WZ}}\right]
\end{aligned}
$$


where $x^{ \pm}=x \pm t$. The phase space of solutions $\mathcal{P}$ can be described by the Cauchy data 1 at $t=0$ :

$$
g(x)=g(0, x) \quad \text { and } \quad \xi_{0}(x)=g^{-1} \partial_{t} g(0, x) .
$$

The corresponding symplectic form is [12]:

$$
\Omega^{\mathrm{bulk}}=\frac{k}{4 \pi} \int_{0}^{2 \pi} \Pi^{G}(g) d x,
$$

where

$$
\Pi^{G}(g)=\operatorname{tr}\left(-\delta \xi_{0} g^{-1} \delta g+\left(\xi_{0}+g^{-1} \partial_{x} g\right)\left(g^{-1} \delta g\right)^{2}\right) .
$$

The $\delta$ denotes here the exterior derivative on the phase space $\mathcal{P}$. It is easy to check that the symplectic form density $\Pi(g)$ has the following exterior derivative

$$
\delta \Pi^{G}(g)=\partial_{x} \omega^{W Z}(g),
$$

what implies closedness of the $\Omega$ :

$$
\delta \Omega^{\text {bulk }}=0 .
$$

The classical equations of motion are

$$
\partial_{-} J_{L}=0 \quad \text { and } \quad \partial_{+} J_{R}=0,
$$

where

$$
J_{L}=-i k \partial_{+} g g^{-1} \text { and } J_{R}=i k g^{-1} \partial_{-} g .
$$

The general solution of (77) satisfying the boundary conditions:

$$
g(t, x+2 \pi)=g(t, x)
$$

is

$$
g(t, x)=g_{L}\left(x^{+}\right) g_{R}^{-1}\left(x^{-}\right)
$$

with $g_{L, R}$ satisfying the monodromy conditions:

$$
\begin{gathered}
g_{L}\left(x^{+}+2 \pi\right)=g_{L}\left(x^{+}\right) \gamma, \\
g_{R}\left(x^{-}+2 \pi\right)=g_{R}\left(x^{-}\right) \gamma
\end{gathered}
$$

\footnotetext{
${ }^{1}$ Surely we can choose any time slice, but for simplicity we always below take the slice $t=0$.
} 
with the same matrix $\gamma$. Expressing the symplectic form density $\Pi^{G}(g)$ in the terms of $g_{L, R}$ we obtain:

$$
\Pi^{G}=\operatorname{tr}\left[g_{L}^{-1} \delta g_{L} \partial_{x}\left(g_{L}^{-1} \delta g_{L}\right)-g_{R}^{-1} \delta g_{R} \partial_{x}\left(g_{R}^{-1} \delta g_{R}\right)+\partial_{x}\left(g_{L}^{-1} \delta g_{L} g_{R}^{-1} \delta g_{R}\right)\right]
$$

Using (13) and (11), (12) one derives for $\Omega^{\text {bulk: }}$

$$
\Omega^{\text {bulk }}=\Omega^{\text {chiral }}\left(g_{L}, \gamma\right)-\Omega^{\text {chiral }}\left(g_{R}, \gamma\right)
$$

where

$$
\Omega^{\text {chiral }}\left(g_{L}, \gamma\right)=\frac{k}{4 \pi} \int_{0}^{2 \pi} \operatorname{tr}\left(g_{L}^{-1} \delta g_{L} \partial_{x}\left(g_{L}^{-1} \delta g_{L}\right)\right) d x+\frac{k}{4 \pi} \operatorname{tr}\left(g_{L}^{-1} \delta g_{L}(0) \delta \gamma \gamma^{-1}\right)
$$

The chiral field $g_{L}$ can be decomposed into the product of a closed loop in $G$, a multivalued field in the Cartan subgroup and a constant element in $G$ :

$$
g_{L}\left(x^{+}\right)=h\left(x^{+}\right) e^{i \tau x^{+} / k} g_{0}^{-1}
$$

where $h \in L G, \tau \in t$ ( the Cartan algebra) and $g_{0} \in G$. For the monodromy of $g_{L}$ we find:

$$
\gamma=g_{0} e^{2 i \pi \tau / k} g_{0}^{-1}
$$

The parametrization (16) induces the following decomposition of $\Omega^{\text {chiral }}\left(g_{L}, \gamma\right)$ :

$$
\Omega^{\text {chiral }}\left(g_{L}, \gamma\right)=\Omega^{L G}(h, \tau)+\frac{k}{4 \pi} \omega_{\tau}(\gamma)+\operatorname{tr}\left[(i \delta \tau) g_{0}^{-1} \delta g_{0}\right]
$$

where $\Omega^{L G}(h, \tau)$ is :

$$
\Omega^{L G}(h, \tau)=\frac{k}{4 \pi} \int_{0}^{2 \pi} \operatorname{tr}\left[h^{-1} \delta h \partial_{x}\left(h^{-1} \delta h\right)+\frac{2 i}{k} \tau\left(h^{-1} \delta h\right)^{2}-\frac{2 i}{k}(\delta \tau) h^{-1} \delta h\right] d x
$$

and $\omega_{\tau}(\gamma)$ is :

$$
\omega_{\tau}(\gamma)=\operatorname{tr}\left[g_{0}^{-1} \delta g_{0} e^{2 i \pi \tau / k} g_{0}^{-1} \delta g_{0} e^{-2 i \pi \tau / k}\right] .
$$

Comparing (14) and (19) to the formulae in appendix $\mathrm{C}$ we see that the symplectic phase space of the WZW model on a cylinder coincides with that of Chern-Simons theory on the annulus times the time-line $\mathcal{A} \times R$. 


\section{Gauged WZW model}

Here we review quantization of the gauged WZW model on the cylinder $\Sigma=$ $R \times S^{1}=(t, x \bmod 2 \pi)$ as it is done in [15].

The action of the gauged WZW model is [2,10,11, 16]:

$$
S^{G / H}(g, A)=S^{\mathrm{WZW}}+S^{\text {gauge }}
$$

where

$$
\begin{gathered}
S^{\text {gauge }}=\frac{k}{2 \pi} \int_{\Sigma} L^{\text {gauge }} \\
L^{\text {gauge }}(g, A)=-\operatorname{tr}\left[-\partial_{+} g g^{-1} A_{-}+g^{-1} \partial_{-} g A_{+}+g A_{+} g^{-1} A_{-}-A_{+} A_{-}\right] .
\end{gathered}
$$

With the help of the Polyakov-Wiegmann identities:

$$
\begin{aligned}
L^{\mathrm{kin}}(g h) & =L^{\mathrm{kin}}(g)+L^{\mathrm{kin}}(h)+\operatorname{Tr}\left(g^{-1} \partial_{z} g \partial_{\bar{z}} h h^{-1}\right)+\operatorname{Tr}\left(g^{-1} \partial_{\bar{z}} g \partial_{z} h h^{-1}\right) \\
\omega^{\mathrm{WZ}}(g h) & =\omega^{\mathrm{WZ}}(g)+\omega^{\mathrm{WZ}}(h)-\mathrm{d}\left(\operatorname{Tr}\left(g^{-1} \mathrm{~d} g \mathrm{~d} h h^{-1}\right)\right)
\end{aligned}
$$

it is easy to check that the action (21) is invariant under the gauge transformation:

$$
g \rightarrow h g h^{-1}, \quad A \rightarrow h A h^{-1}-d h h^{-1}
$$

for $h: \Sigma \rightarrow H$.

The equations of motions are:

$$
D_{+}\left(g^{-1} D_{-} g\right)=0, \quad \operatorname{Tr}\left(g^{-1} D_{-} g T_{H}\right)=\operatorname{Tr}\left(g D_{+} g^{-1} T_{H}\right)=0, \quad F(A)=0,
$$

where $D_{ \pm} g=\partial_{ \pm} g+\left[A_{ \pm}, g\right]$ and $T_{H}$ is any element in the $H$ Lie algebra.

The flat gauge field $A$ can be written as $h^{-1} d h$ for $h: R^{2} \rightarrow H$ and satisfying:

$$
h(t, x+2 \pi)=\rho^{-1} h(t, x)
$$

for some $\rho \in H$.

Define $\tilde{g}=h g h^{-1}$. Note that $\tilde{g}$ satisfies

$$
\tilde{g}(t, x+2 \pi)=\rho^{-1} \tilde{g}(t, x) \rho .
$$

In the terms of $\tilde{g}$ equations (27) take the form:

$$
\partial_{+}\left(\tilde{g}^{-1} \partial_{-} \tilde{g}\right)=0, \quad \operatorname{Tr}\left(\tilde{g}^{-1} \partial_{-} \tilde{g} T_{H}\right)=\operatorname{Tr}\left(\tilde{g} \partial_{+} \tilde{g}^{-1} T_{H}\right)=0
$$


The canonical symplectic form density, obtained following the general prescription [4,5, 12, is given by:

$$
\Pi^{G / H}(g, h)=\Pi^{G}(\tilde{g})+\partial_{x} \Psi(h, g),
$$

where

$$
\Psi(h, g)=\operatorname{tr} h^{-1} d h\left(g^{-1} d g+d g g^{-1}+g^{-1} h^{-1} d h g\right) .
$$

Some properties of the form (32) are summarized in appendix A.

Integrating (31) we get the canonical symplectic form:

$$
\Omega^{G / H}=\frac{k}{4 \pi} \int_{0}^{2 \pi} \Pi^{G}(\tilde{g}) d x+\frac{k}{4 \pi} \Psi\left(\rho^{-1}, h g h^{-1}(0)\right) .
$$

Collecting (5), (29) and (92) one can show that the form (33) is closed.

Equations (30) can be solved in the terms of the chiral fields:

$$
\tilde{g}=g_{L}\left(x^{+}\right) g_{R}^{-1}\left(x^{-}\right), \quad \operatorname{Tr}\left(\partial_{y} g_{L} g_{L}^{-1} T_{H}\right)=\operatorname{Tr}\left(\partial_{y} g_{R} g_{R}^{-1} T_{H}\right)=0
$$

with the monodromy properties:

$$
g_{L}(y+2 \pi)=\rho^{-1} g_{L}(y) \gamma, \quad g_{R}(y+2 \pi)=\rho^{-1} g_{R}(y) \gamma .
$$

The monodromy properties (35) imply that the chiral fields $g_{L, R}$ should be written as products of fields as well:

$$
g_{L}=h_{B}^{-1} g_{A}, \quad g_{R}=h_{D}^{-1} g_{C},
$$

where $h_{B}, h_{D} \in H$ and $g_{A}, g_{C} \in G$. The fields in (36) should additionally satisfy:

$$
\operatorname{tr}\left[T_{H}\left(\partial_{y} h_{B} h_{B}^{-1}-\partial_{y} g_{A} g_{A}^{-1}\right)\right]=0, \quad \operatorname{tr}\left[T_{H}\left(\partial_{y} h_{D} h_{D}^{-1}-\partial_{y} g_{C} g_{C}^{-1}\right)\right]=0
$$

and

$$
\begin{array}{ll}
h_{B}(y+2 \pi)=h_{B}(y) \rho, & g_{A}(y+2 \pi)=g_{A}(y) \gamma, \\
h_{D}(y+2 \pi)=h_{D}(y) \rho, & g_{C}(y+2 \pi)=g_{C}(y) \gamma .
\end{array}
$$

Using (37) one can show:

$$
\operatorname{tr}\left[g_{L}^{-1} \delta g_{L} \partial_{y}\left(g_{L}^{-1} \delta g_{L}\right)\right]=\operatorname{tr}\left[g_{A}^{-1} \delta g_{A} \partial_{y}\left(g_{A}^{-1} \delta g_{A}\right)-h_{B}^{-1} \delta h_{B} \partial_{y}\left(h_{B}^{-1} \delta h_{B}\right)+\partial_{y}\left(\delta h_{B} h_{B}^{-1} \delta g_{A} g_{A}^{-1}\right)\right]
$$

and similarly for $g_{R}$ and $h_{D}, g_{C}$. 
Collecting (34), (35), (36), (38), (39), (40) and (13) one can show that

$$
\Omega^{G / H}=\Omega^{\text {chiral }}\left(g_{A}, \gamma\right)-\Omega^{\text {chiral }}\left(g_{C}, \gamma\right)-\Omega^{\text {chiral }}\left(h_{B}, \rho\right)+\Omega^{\text {chiral }}\left(h_{D}, \rho\right)
$$

Comparing (41) with (14) and remembering that the latter is the symplectic form of the Chern-Simons theory on $\mathcal{A} \times R$, we arrive at the conclusion that the phase space of the gauged WZW model on a cylinder coincides with that of double Chern-Simons theory [15, 17] on $\mathcal{A} \times R$.

\section{Permutation branes}

Worldvolume $Q$ of the permutation branes on product of cosets $G / H \times G / H$ corresponding to a primary $(\mu, \nu)$ has been constructed in [18] and have the form:

$$
\left(g_{1}, g_{2}\right)=\left(\operatorname{cbp}, L p^{-1} L^{-1}\right),
$$

where $p \in G, L \in H, c \in C_{H}^{\nu}, b \in C_{G}^{\mu}$ and $C_{G}^{\mu}$ are the conjugacy classes in $G$ :

$$
C_{G}^{\mu}=\left\{\beta f_{\mu} \beta^{-1}=\beta e^{2 i \pi \mu / k} \beta^{-1}, \quad \beta \in G\right\},
$$

where $\boldsymbol{\mu} \equiv \boldsymbol{\mu} \cdot \boldsymbol{H}$ is a highest weight representation integrable at level $k$, taking value in the Cartan subalgebra of the $G$ Lie algebra. $C_{H}^{\nu}$ are the similarly defined conjugacy classes in $H$. If $G$ and $H$ possess common center, $\mu$ and $\nu$ should satisfy the selection rule [8].

To write the action one should introduce an auxiliary disc $D$ satisfying the condition $\partial B=\Sigma+D$ and continue the fields $g_{1}$ and $g_{2}$ on this disc always holding the condition (42).

The action with the boundary condition (42) has the form

$$
S_{\mathcal{P}}^{G / H \times G / H}=S^{G / H}\left(g_{1}, A_{1}\right)+S^{G / H}\left(g_{2}, A_{2}\right)-\frac{k}{4 \pi} \int_{D} \varpi(L, p, c, b)
$$

where

$$
\varpi(L, p, c, b)=\Omega^{(2)}(c, b)-\operatorname{tr}\left((c b)^{-1} d(c b) d p p^{-1}\right)+\Psi(L, p),
$$

where

$$
\Omega^{(2)}(c, b)=\omega_{\nu}(c)-\operatorname{tr}\left(c^{-1} d c d b b^{-1}\right)+\omega_{\mu}(b)
$$

and $\omega_{\mu}(C)$ is defined in (20) and $\Psi(L, p)$ is defined in (32). The form $\varpi(L, p, c, b)$ satisfies the condition:

$$
d \varpi(L, p, c, b)=\left.\omega^{W Z}\left(g_{1}\right)\right|_{Q}+\left.\omega^{W Z}\left(g_{2}\right)\right|_{Q} .
$$


One can check that the action (44) is invariant under the gauge transformations:

$$
\begin{aligned}
& g_{1} \rightarrow h_{1} g_{1} h_{1}^{-1}, \quad A_{1} \rightarrow h_{1} A_{1} h_{1}^{-1}-d h_{1} h_{1}^{-1}, \\
& g_{2} \rightarrow h_{2} g_{2} h_{2}^{-1}, \quad A_{2} \rightarrow h_{2} A_{2} h_{2}^{-1}-d h_{2} h_{2}^{-1},
\end{aligned}
$$

where $h_{1}: \Sigma \rightarrow H, h_{2}: \Sigma \rightarrow H$. For this purpose note that under (48) the boundary parameters transform in the following way:

$$
p \rightarrow h_{1} p h_{1}^{-1}, \quad c \rightarrow h_{1} c h_{1}^{-1}, \quad b \rightarrow h_{1} b h_{1}^{-1}, \quad L \rightarrow h_{2} L h_{1}^{-1}
$$

The gauge invariance follows from the Polyakov-Wiegmann identities and the transformation properties of $\varpi(L, p, c, b)$ :

$\varpi\left(h_{2} L h_{1}^{-1}, h_{1} p h_{1}^{-1}, h_{1} c h_{1}^{-1}, h_{1} b h_{1}^{-1}\right)-\varpi(L, p, c, b),=-\Psi\left(h_{1}, c b p\right)-\Psi\left(h_{2}, L p^{-1} L^{-1}\right)$

which can be obtained using formulae of appendix A.

Consider $G / H \times G / H$ product of coset models on the strip $R \times[0, \pi]$ with boundary conditions on both sides given by the permutation branes:

$$
\begin{aligned}
& \left(g_{1}, g_{2}\right)(0)=\left(C_{2} C_{1} p_{1}, L_{1} p_{1}^{-1} L_{1}^{-1}\right) \\
& \left(g_{1}, g_{2}\right)(\pi)=\left(C_{4} C_{3} p_{2}, L_{2} p_{2}^{-1} L_{2}^{-1}\right)
\end{aligned}
$$

Here $C_{1} \in C_{G}^{\mu_{1}}, C_{2} \in C_{H}^{\mu_{2}}, C_{3} \in C_{G}^{\mu_{3}}, C_{4} \in C_{H}^{\mu_{4}}, L_{1}, L_{2} \in H, p_{1}, p_{2} \in G$.

The boundary equation of motion resulting from the action (44) at $x=0$ are:

$$
\begin{gathered}
g_{1}^{-1} D_{-} g_{1}+L_{1}^{-1} g_{2} D_{+} g_{2}^{-1} L_{1}=0 \\
C_{2}^{-1} g_{1} D_{+} g_{1}^{-1} C_{2}+L_{1}^{-1} g_{2}^{-1} D_{-} g_{2} L_{1}=0 \\
L_{1}^{-1} D_{t} L_{1}=0 \quad C_{2}^{-1} D_{t} C_{2}=0
\end{gathered}
$$

where $D_{t}=D_{+}-D_{-}, D_{ \pm} L=\partial_{ \pm} L+A_{2 \pm} L-L A_{1 \pm}, D_{ \pm} g_{1}=\partial_{ \pm} g_{1}+\left[A_{1 \pm}, g_{1}\right]$, $D_{ \pm} g_{2}=\partial_{ \pm} g_{2}+\left[A_{2 \pm}, g_{2}\right], D_{ \pm} C_{2}=\partial_{ \pm} C_{2}+\left[A_{1 \pm}, C_{2}\right]$.

Derivation of the equations (53), (54), (55) is outlined in the appendix B. Parameterising again flat gauge fields as

$$
A_{1}=h_{1}^{-1} d h_{1} \quad A_{2}=h_{2}^{-1} d h_{2}
$$


one can define as before

$$
\begin{array}{cc}
\tilde{g}_{1}=h_{1} g_{1} h_{1}^{-1} & \tilde{g}_{2}=h_{2} g_{2} h_{2}^{-1} \\
\tilde{C}_{1}=h_{1} C_{1} h_{1}^{-1} & \tilde{C}_{2}=h_{1} C_{2} h_{1}^{-1} \\
\tilde{p}_{1}=h_{1} p_{1} h_{1}^{-1} & \tilde{L}_{1}=h_{2} L_{1} h_{1}^{-1} \\
\tilde{C}_{3}=h_{1} C_{3} h_{1}^{-1} & \tilde{C}_{4}=h_{1} C_{4} h_{1}^{-1} \\
\tilde{p}_{2}=h_{1} p_{2} h_{1}^{-1} & \tilde{L}_{2}=h_{2} L_{2} h_{1}^{-1}
\end{array}
$$

and we have the bulk equations (30) for $\tilde{g}_{1}$ and $\tilde{g}_{2}$ and boundary equations take the form:

$$
\begin{gathered}
\tilde{g}_{1}^{-1} \partial_{-} \tilde{g}_{1}+\tilde{L}_{1}^{-1} \tilde{g}_{2} \partial_{+} \tilde{g}_{2}^{-1} \tilde{L}_{1}=0 \\
\tilde{C}_{2}^{-1} \tilde{g}_{1} \partial_{+} \tilde{g}_{1}^{-1} \tilde{C}_{2}+\tilde{L}_{1}^{-1} \tilde{g}_{2}^{-1} \partial_{-} \tilde{g}_{2} \tilde{L}_{1}=0 \\
\tilde{L}_{1}^{-1} \partial_{t} \tilde{L}_{1}=0 \quad \tilde{C}_{2}^{-1} \partial_{t} \tilde{C}_{2}=0
\end{gathered}
$$

Equation (60) implies that $\tilde{L}_{1}$ and $\tilde{C}_{2}$ are constant along the boundary. Boundary conditions (51) and (52) imply

$$
\begin{aligned}
& \left(\tilde{g}_{1}, \tilde{g}_{2}\right)(0)=\left(\tilde{C}_{2} \tilde{C}_{1} \tilde{p}_{1}, \tilde{L}_{1} \tilde{p}_{1}^{-1} \tilde{L}_{1}^{-1}\right) \\
& \left(\tilde{g}_{1}, \tilde{g}_{2}\right)(\pi)=\left(\tilde{C}_{4} \tilde{C}_{3} \tilde{p}_{2}, \tilde{L}_{2} \tilde{p}_{2}^{-1} \tilde{L}_{2}^{-1}\right)
\end{aligned}
$$

Using the chiral decomposition one can solve the boundary equation of motion

$$
\begin{gathered}
g_{1 R}(y)=\tilde{L}_{1}^{-1} g_{2 L}(-y) m \\
g_{2 R}(y)=\tilde{L}_{1} \tilde{C}_{2}^{-1} g_{1 L}(-y) n
\end{gathered}
$$

Equations (63) and (64) indeed imply (61) with

$$
\begin{gathered}
\tilde{p}_{1}(t)=\tilde{C}_{2}^{-1} g_{1 L}(t) n g_{2 L}^{-1}(t) \tilde{L}_{1} \\
\tilde{C}_{1}=\tilde{C}_{2}^{-1} g_{1 L}(t) m^{-1} n^{-1} g_{1 L}^{-1}(t) \tilde{C}_{2}
\end{gathered}
$$

To have that $\tilde{C}_{1} \in C_{G}^{\mu_{1}}$ we should require $m^{-1} n^{-1} \equiv R_{0} \in C_{G}^{\mu_{1}}$.

To satisfy (62) we assume the following monodromy properties of $g_{1 L}$ and $g_{2 L}$

$$
g_{1 L}(y+2 \pi)=\rho_{1}^{-1} g_{1 L}(y) \gamma_{1} \quad g_{2 L}(y+2 \pi)=\rho_{2}^{-1} g_{2 L}(y) \gamma_{2}
$$

Now one can show that (62) is satisfied with

$$
\tilde{p}_{2}(t)=\tilde{L}_{2}^{-1} \tilde{L}_{1} \tilde{C}_{2}^{-1} \rho_{1} g_{1 L}(\pi+t) \gamma_{1}^{-1} n g_{2 L}^{-1}(\pi+t) \tilde{L}_{2}
$$




$$
\tilde{C}_{3}=\tilde{C}_{4}^{-1} g_{1 L}(\pi+t) m^{-1} \gamma_{2} n^{-1} \gamma_{1}\left(\tilde{C}_{4}^{-1} g_{1 L}(\pi+t)\right)^{-1}
$$

if we require

$$
\rho_{2}^{-1}=\tilde{L}_{2} \tilde{L}_{1}^{-1}
$$

and

$$
\rho_{1}^{-1} \tilde{C}_{2} \tilde{L}_{1}^{-1} \tilde{L}_{2}=\rho_{1}^{-1} \tilde{C}_{2} \tilde{\rho}_{2}^{-1}=\tilde{C}_{4}
$$

where $\tilde{\rho}_{2}=\tilde{L}_{1}^{-1} \rho_{2} \tilde{L}_{1}$.

To have that $\tilde{C}_{3} \in C_{G}^{\mu_{3}}$ we should require $m^{-1} \gamma_{2} n^{-1} \gamma_{1}=\tilde{\gamma}_{2} R_{0} \gamma_{1}=R_{\pi} \in C_{G}^{\mu_{3}}$, where $\tilde{\gamma}_{2}=m^{-1} \gamma_{2} m$.

The monodromies (67) as before can be realized in the terms of the decomposition of the fields $g_{1 L}$ and $g_{2 L}$ as products:

$$
g_{1 L}=h_{B}^{-1} g_{A}, \quad g_{2 L}=h_{D}^{-1} g_{C}
$$

of the new fields $h_{B}, g_{A}, h_{D}, g_{C}$ possessing the monodromy properties:

$$
\begin{array}{ll}
h_{B}(2 \pi)=h_{B}(0) \rho_{1}, & g_{A}(2 \pi)=g_{A}(0) \gamma_{1}, \\
h_{D}(2 \pi)=h_{D}(0) \rho_{2}, & g_{C}(2 \pi)=g_{C}(0) \gamma_{2},
\end{array}
$$

and satisfying (37).

The symplectic form of product of the gauged WZW models on the strip with boundary conditions specified by the permutation branes can be written using the symplectic form density (31) and the form $\varpi$ :

$\Omega_{\mathcal{P}}^{G / H}=\frac{k}{4 \pi}\left[\int_{0}^{\pi} \Pi^{G / H}\left(g_{1}, h_{1}\right) d x+\int_{0}^{\pi} \Pi^{G / H}\left(g_{2}, h_{2}\right) d x+\varpi\left(g_{1}(0), g_{2}(0)\right)-\varpi\left(g_{1}(\pi), g_{2}(\pi)\right)\right]$.

Substituting in (75) the symplectic form density (31) and using the transformation property (50) we obtain:

$\Omega_{\mathcal{P}}^{G / H}=\frac{k}{4 \pi}\left[\int_{0}^{\pi} \Pi\left(\tilde{g}_{1}\right) d x+\int_{0}^{\pi} \Pi\left(\tilde{g}_{2}\right) d x+\varpi\left(\tilde{L}_{1}, \tilde{p}_{1}, \tilde{C}_{2}, \tilde{C}_{1}\right)-\varpi\left(\tilde{L}_{2}, \tilde{p}_{2}, \tilde{C}_{4}, \tilde{C}_{3}\right)\right]$,

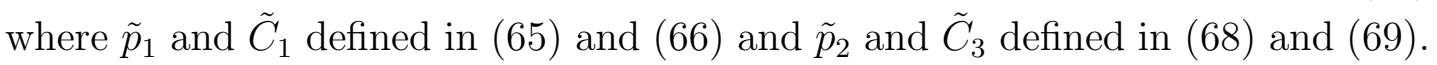

Using (16) one can obtain for (76):

$$
\Omega_{\mathcal{P}}^{G / H}=\Omega^{L G}\left(s_{1}, \tau_{1}\right)+\Omega^{L G}\left(s_{2}, \tau_{2}\right)-\Omega^{L G}\left(s_{3}, \tau_{3}\right)-\Omega^{L G}\left(s_{4}, \tau_{4}\right)+\Omega_{1}^{\text {bndry }}-\Omega_{2}^{\text {bndry }}
$$




$$
\begin{array}{r}
\Omega_{1}^{\text {bndry }}=\operatorname{tr}\left[\left(i \delta \tau_{1}\right) f_{1}^{-1} \delta f_{1}\right]+\operatorname{tr}\left[\left(i \delta \tau_{2}\right) f_{2}^{-1} \delta f_{2}\right] \\
+\frac{k}{4 \pi}\left[\omega_{\tau_{1}}\left(\gamma_{1}\right)+\omega_{\tau_{2}}\left(\tilde{\gamma}_{2}\right)+\omega_{\mu_{1}}\left(R_{0}\right)-\omega_{\mu_{3}}\left(R_{\pi}\right)\right. \\
\left.-\operatorname{tr}\left(R_{0}^{-1} \delta R_{0} \delta \gamma_{1} \gamma_{1}^{-1}\right)-\operatorname{tr}\left(\tilde{\gamma}_{2}^{-1} \delta \tilde{\gamma}_{2} \delta R_{0} R_{0}^{-1}\right)-\operatorname{tr}\left(R_{0}^{-1} \tilde{\gamma}_{2}^{-1} \delta \tilde{\gamma}_{2} R_{0} \delta \gamma_{1} \gamma_{1}^{-1}\right)\right] \\
\Omega_{2}^{\text {bndry }}=\operatorname{tr}\left[\left(i \delta \tau_{3}\right) f_{3}^{-1} \delta f_{3}\right]+\operatorname{tr}\left[\left(i \delta \tau_{4}\right) f_{4}^{-1} \delta f_{4}\right] \\
+\frac{k}{4 \pi}\left[\omega_{\tau_{3}}\left(\rho_{1}\right)+\omega_{\tau_{4}}\left(\tilde{\rho}_{2}\right)-\omega_{\mu_{2}}\left(\tilde{C}_{2}\right)+\omega_{\mu_{4}}\left(\tilde{C}_{4}\right)\right. \\
\left.+\operatorname{tr}\left(\delta \tilde{C}_{2} \tilde{C}_{2}^{-1} \delta \rho_{1} \rho_{1}^{-1}\right)+\operatorname{tr}\left(\tilde{\rho}_{2}^{-1} \delta \tilde{\rho}_{2} \tilde{C}_{2}^{-1} \delta \tilde{C}_{2}\right)-\operatorname{tr}\left(\tilde{C}_{2} \tilde{\rho}_{2}^{-1} \delta \tilde{\rho}_{2} \tilde{C}_{2}^{-1} \delta \rho_{1} \rho_{1}^{-1}\right)\right]
\end{array}
$$

Comparing (77) with the corresponding formulae in appendix $\mathrm{C}$ we arrive at the conclusion that the phase space of product of coset models on a strip with boundary conditions specified by permutation branes is symplectomorphic to the phase space of the double Chern-Simons theory on an annulus times the time-line and with $G$ and $H$ gauge fields both coupled to two Wilson lines.

\section{Permutation branes in topological G/G coset}

In this section we discuss permutation branes on the product of topological coset $G / G \times G / G$.

In the previous section we have seen that the phase space of the product of the gauged WZW models on a strip with boundary conditions given by the permutation branes is symplectomorphic to the phase space of the double ChernSimons theory on an annulus times the time-line with $G$ and $H$ gauge fields both coupled to two Wilson lines. In the case when $G=H$ we arrive at the conclusion that product of topological cosets $G / G \times G / G$ on a strip with boundary conditions given by the permutation branes is equivalent to the Chern-Simons theory on the torus $T^{2}=\mathcal{A} \cup(-\mathcal{A})$ times the time-line $R$ with four Wilson lines. This can be verified by a direct calculation. For the case $G=H$ the bulk equations of motion (30) imply that $\tilde{g}_{1}$ and $\tilde{g}_{2}$ are $(t, x)$ independent.

Therefore one has:

$$
\begin{gathered}
\tilde{g}_{1}(0)=\tilde{C}_{2} \tilde{C}_{1} \tilde{p}_{1}=\tilde{g}_{1}(\pi)=\tilde{C}_{4} \tilde{C}_{3} \tilde{p}_{2} \\
\tilde{g}_{2}(0)=\tilde{L}_{1} \tilde{p}_{1}^{-1} \tilde{L}_{1}^{-1}=\tilde{g}_{2}(\pi)=\tilde{L}_{2} \tilde{p}_{2}^{-1} \tilde{L}_{2}^{-1}
\end{gathered}
$$

From equations (80) and (81) we get 


$$
m \tilde{p}_{2} m^{-1} \tilde{p}_{2}^{-1} \tilde{C}_{3}^{-1} \tilde{C}_{4}^{-1} \tilde{C}_{2} \tilde{C}_{1}=1
$$

where

$$
m=\tilde{L}_{1}^{-1} \tilde{L}_{2}
$$

The symplectic form (76) in this case reduces to

$$
\Omega_{\mathcal{P}}^{G / G}=\frac{k}{4 \pi}\left[\varpi\left(\tilde{L}_{1}, \tilde{p}_{1}, \tilde{C}_{2}, \tilde{C}_{1}\right)-\varpi\left(\tilde{L}_{2}, \tilde{p}_{2}, \tilde{C}_{4}, \tilde{C}_{3}\right)\right]
$$

Comparing formulae (82) and (84) with the corresponding formulae in appendix $\mathrm{C}$ we arrive at the mentioned symplectomorphism of the product of topological cosets $G / G \times G / G$ on a strip with the boundary conditions given by the permutation branes and that of Chern-Simons theory on the torus times the time-line with four Wilson lines.

\section{Final Remarks}

The constructions in section 4 and 5 can be easily generalized to $N$-fold product of coset models $G / H$. The ansatz for permutation branes has the form:

$$
\left(g_{1}, \ldots, g_{N}\right)=\left(C_{2} C_{1} p_{N-1} \cdots p_{1}, L_{1} p_{1}^{-1} L_{1}^{-1}, \ldots, L_{N-1} p_{N-1}^{-1} L_{N-1}^{-1}\right)
$$

where $C_{1} \in C_{G}^{\mu_{1}}, C_{2} \in C_{H}^{\mu_{2}}, p_{i} \in G, L_{i} \in H$. The ansatz is invariant under the $N$-fold adjoint action $: g_{i} \rightarrow h_{i} g_{i} h_{i}^{-1}$, where $h_{i}: \Sigma \rightarrow H$. Using the PolyakovWiegmann identity (25) it is straightforward to check the existence of the twoform $\varpi_{N}$ satisfying the relation:

$$
\left.\sum_{1}^{N} \omega^{\mathrm{WZ}}\left(g_{i}\right)\right|_{\text {brane }}=d \varpi_{N}
$$

Performing the same steps as before we arrive at the conclusion, that the phase space of the $N$-fold product of the gauged WZW model $G / H$ on a strip with boundary conditions given by permutation branes is symplectomorphic to the phase space of the double Chern-Simons theory on a sphere with $N$ holes times the time-line and with $G$ and $H$ gauge fields both coupled to two Wilson lines. For the special case of the toplogical coset $G / G$ we get, that the phase space of the $N$-fold product of the topological cosets $G / G$ on a strip with boundary 
conditions given by permutation branes is symplectomorphic to the phase space of Chern-Simons theory on a Riemann surface of genus $N-1$ times the time-line with four Wilson lines. 


\section{A Useful formulae}

In this appendix we collect some useful properties of the two-form $\Psi(h, g)$ defined by formula (32).

$$
\begin{gathered}
\Psi(h L, p)=\Psi(L, p)+\Psi\left(h, L p L^{-1}\right) . \\
\Psi\left(L h^{-1}, h p h^{-1}\right)=\Psi(L, p)-\Psi(h, p) . \\
\omega_{\mu}\left(h C h^{-1}\right)-\omega_{\mu}(C)=-\Psi(h, C) . \\
\Omega^{(2)}\left(h C_{1} h^{-1}, h C_{2} h^{-1}\right)-\Omega^{(2)}\left(C_{1}, C_{2}\right)=-\Psi\left(h, C_{1} C_{2}\right), \\
\Psi\left(h, C_{1} C_{2}\right)=\Psi\left(h, C_{1}\right)+\Psi\left(h, C_{2}\right)+\left(\tilde{C}_{1}^{-1} d \tilde{C}_{1} d \tilde{C}_{2} \tilde{C}_{2}^{-1}-C_{1}^{-1} d C_{1} d C_{2} C_{2}^{-1}\right),
\end{gathered}
$$

where $\tilde{C}_{1}=h C_{1} h^{-1}$ and $\tilde{C}_{2}=h C_{2} h^{-1}$.

$$
\omega^{W Z W}\left(h g h^{-1}\right)-\omega^{W Z W}(g)=-d \Psi(h, g) .
$$

\section{B Boundary Equations of motion}

Computing variation of the action (44) one obtains for the boundary part:

$$
\begin{array}{r}
\operatorname{tr}\left[g_{1}^{-1} \delta g_{1}\left(g_{1}^{-1} \partial_{+} g_{1}+g_{1}^{-1} \partial_{-} g_{1}\right)\right] d t+\operatorname{tr}\left[g_{2}^{-1} \delta g_{2}\left(g^{-1} \partial_{+} g_{2}+g_{2}^{-1} \partial_{-} g\right)\right] d t \\
+2 \operatorname{tr}\left[\delta g_{1} g_{1}^{-1} A_{1-}-A_{1+} g_{1}^{-1} \delta g_{1}+\left(\delta g_{2} g_{2}^{-1} A_{2-}-A_{2+} g_{2}^{-1} \delta g_{2}\right)\right] d t+B=0 .
\end{array}
$$

The last term $B$ is one-form satisfying the relation:

$$
\operatorname{tr}\left(g_{1}^{-1} \delta g_{1}\left(g_{1}^{-1} d g_{1}\right)^{2}\right)+\operatorname{tr}\left(g_{2}^{-1} \delta g_{2}\left(g_{2}^{-1} d g_{2}\right)^{2}\right)-\delta \varpi=d B .
$$

Recalling that the first two terms come from the equation

$$
\delta \omega^{W Z}=d\left[\operatorname{tr}\left(g^{-1} \delta g\left(g^{-1} d g\right)^{2}\right)\right],
$$

we see that the existence of the one-form $B$ satisfying (94) is a consequence of the equation (47). Using (42) one can compute $B$ explicitly:

$$
\begin{aligned}
& B=A_{\mu_{1}}\left(C_{1}\right)+A_{\mu_{2}}\left(C_{2}\right)+\operatorname{tr}\left[C_{2}^{-1} \delta C_{2} d C_{1} C_{1}^{-1}-\delta C_{1} C_{1}^{-1} C_{2}^{-1} d C_{2}-\right. \\
& \delta p p^{-1}\left(C_{2} C_{1}\right)^{-1} d\left(C_{2} C_{1}\right)+\left(C_{2} C_{1}\right)^{-1} \delta\left(C_{2} C_{1}\right) d p p^{-1}-L^{-1} \delta L p^{-1} d p+ \\
& p^{-1} \delta p L^{-1} d L-L^{-1} \delta L d p p^{-1}+\delta p p^{-1} L^{-1} d L-L^{-1} \delta L p^{-1} L^{-1} d L p+ \\
& \left.L^{-1} \delta L p L^{-1} d L p^{-1}\right] .
\end{aligned}
$$


The one-form $A_{\mu}(C)$ was defined in [7]:

$$
A_{\mu}(C)=\operatorname{tr}\left[h^{-1} \delta h\left(f_{\mu}^{-1} h^{-1} d h f_{\mu}-f_{\mu} h^{-1} d h f_{\mu}^{-1}\right)\right],
$$

where $C=h f_{\mu} h^{-1}, f_{\mu}=e^{2 \pi i \mu / k}$,

and satisfies:

$$
\left.\operatorname{tr}\left(g^{-1} \delta g\left(g^{-1} d g\right)^{2}\right)\right|_{g=C}-\delta \omega_{\mu}(C)=d A_{\mu}(C) .
$$

$A_{\mu}(C)$ satisfies also another important relation on the time-line:

$$
\operatorname{tr}\left[g^{-1} \delta g\left(g^{-1} \partial_{+} g+g^{-1} \partial_{-} g\right)\right] d t+A_{\mu}(C)=\operatorname{tr}\left[2 \delta h h^{-1}\left(\partial_{+} g g^{-1}-g^{-1} \partial_{-} g\right)\right] d t,
$$

where $g=C$. Let us explain the meaning of this equation.

The left hand side of the (99) is a particular case of (93) and describes boundary equation of motion of the WZW model with the boundary condition specified by the conjugacy class $C$, while the right hand side proportional to $J_{L}+J_{R}$, what is the condition for the diagonal chiral algebra preservation.

Now, using (42) and (96), one can show by a straightforwatd calculation, that (93) implies the equations (53), (54), (55) in section 4.

\section{Symplectic forms of the moduli space of flat connections on a Riemann surface}

In this appendix we briefly review the symplectic phase space of the Chern-Simons theory on the three-dimensional manifold of the form $M \times R$, where $M$ is twodimensional Riemann surface, $R$ is time direction, with $n$ time-like Wilson lines assigned with representations $\lambda_{i}$. It was shown in [6,22] that the phase space of the Chern-Simons theory in such a situation is given by the moduli space of flat connections on the Riemann surface $M$ punctured at the points $z_{i}$ where Wilson lines hit $M$, with the holonomies around punctures belonging to the conjugacy classes $C_{G}^{\lambda_{i}}=\eta e^{2 \pi i \lambda_{i} / k} \eta^{-1}$. Therefore denoting holonomies around handles $a_{j}$ and $b_{j}$ by $A_{j}$ and $B_{j}$, and around punctures by $M_{i} \in C_{G}^{\lambda_{i}}$ we arrive at the conclusion that the phase space of the Chern-Simons theory is

$$
\mathcal{F}_{g, n}=G^{2 g} \times \prod_{i=1}^{n} C_{G}^{\lambda_{i}}
$$


subject to the relation

$$
\left[B_{g}, A_{g}^{-1}\right] \cdots\left[B_{1}, A_{1}^{-1}\right] M_{n} \cdots M_{1}=I
$$

where

$$
\left[B_{j}, A_{j}\right]=B_{j} A_{j} B_{j}^{-1} A_{j}^{-1}
$$

and to the adjoint group action.

The symplectic form on $\mathcal{F}_{g, n}$ was derived in [1] and has the form:

$$
\Omega_{\mathcal{M}_{g, n}}=\sum_{i=1}^{n} \Omega_{M_{i}}+\sum_{j=1}^{g} \Omega_{H_{j}},
$$

where

$$
\begin{gathered}
\Omega_{M_{i}}=\frac{k}{4 \pi} \omega_{\lambda_{i}}\left(M_{i}\right)+\frac{k}{4 \pi} \operatorname{tr}\left(K_{i-1}^{-1} \delta K_{i-1} K_{i}^{-1} \delta K_{i}\right) \\
\Omega_{H_{j}}=\frac{k}{4 \pi} \Psi\left(B_{j}, A_{j}\right)+\frac{k}{4 \pi}\left(\operatorname{tr}\left(K_{n+2 j-2}^{-1} \delta K_{n+2 j-2} K_{n+2 j-1}^{-1} \delta K_{n+2 j-1}\right)\right. \\
\left.+\operatorname{tr}\left(K_{n+2 j-1}^{-1} \delta K_{n+2 j-1} K_{n+2 j}^{-1} \delta K_{n+2 j}\right)\right),
\end{gathered}
$$

and where

$$
\begin{gathered}
K_{i}=M_{i} \cdots M_{1} \quad i \leq n, \\
K_{n+2 j-1}=A_{j}\left[B_{j-1}, A_{j-1}^{-1}\right] \cdots\left[B_{1}, A_{1}^{-1}\right] K_{n}, \\
K_{n+2 j}=\left[B_{j}, A_{j}^{-1}\right] \cdots\left[B_{1}, A_{1}^{-1}\right] K_{n} \quad 1 \leq j \leq g .
\end{gathered}
$$

$K_{0}$ can be chosen to be equal to the unity element. According to (101) also

$$
K_{n+2 g}=I \text {. }
$$

$\omega_{\lambda}(M)$ and $\Psi(B, A)$ are defined in equations (20) and (32) correspondingly.

It was also proved in [1] that quantization of the moduli space $\mathcal{F}_{g, n}$ with the symplectic form (103) leads to the space of $n$-point conformal blocks on a Riemann surface of the genus $g$.

The last piece of information which we need is the symplectic form on the moduli space of flat connections on the punctured sphere with holes $S_{n . m}^{2}$, where $n$ as before is number of punctures and $m$ is number of holes. It was argued in [6, 14] that the corresponding symplectic form $\Omega_{S_{n, m}^{2}}$ is given by:

$$
\Omega_{S_{n, m}^{2}}=\Omega_{S_{n+m, 0}^{2}}+\sum_{i=1}^{m} \Omega_{i}^{\mathrm{LG}},
$$

where $\Omega^{\mathrm{LG}}$ is defined in (19) and its geometrical quantization leads to the integrable representation of the affine algebra $\hat{g}$ at level $k$. 


\section{References}

[1] A. Y. Alekseev and A. Z. Malkin, "Symplectic structure of the moduli space of flat connection on a Riemann surface," Commun. Math. Phys. 169 (1995) 99 arXiv:hep-th/9312004.

[2] K. Bardakci, E. Rabinovici and B. Saering, "String Models With $C<1$ Components," Nucl. Phys. B 299 (1988) 151.

[3] M. F. Chu, P. Goddard, I. Halliday, D. I. Olive and A. Schwimmer, "Quantization Of The Wess-Zumino-Witten Model On A Circle," Phys. Lett. B 266 (1991) 71.

[4] C. Crnkovic, "Symplectic geometry of the covariant phase space, superstrings and superspace," Class. Quant. Grav. 5 (1988) 1557.

[5] C. Crnkovic and E. Witten, "Covariant description of canonical formalism in geometrical theories," in *Hawking, S.W. (ed.), Israel, W. (ed.): Three hundred years of gravitation*, 676-684.

[6] S. Elitzur, G. W. Moore, A. Schwimmer and N. Seiberg, "Remarks On The Canonical Quantization Of The Chern-Simons-Witten Theory," Nucl. Phys. B 326 (1989) 108.

[7] S. Elitzur, A. Giveon, D. Kutasov, E. Rabinovici and G. Sarkissian, "Dbranes in the background of NS fivebranes," JHEP 0008 (2000) 046 arXiv:hep-th/0005052.

[8] S. Elitzur and G. Sarkissian, "D-branes on a gauged WZW model," Nucl. Phys. B 625 (2002) 166 arXiv:hep-th/0108142].

[9] F. Falceto and K. Gawedzki, "Lattice Wess-Zumino-Witten Model And Quantum Groups," J. Geom. Phys. 11 (1993) 251 arXiv:hep-th/9209076.

[10] K. Gawedzki and A. Kupiainen, "G/h Conformal Field Theory from Gauged WZW Model," Phys. Lett. B 215 (1988) 119.

[11] K. Gawedzki and A. Kupiainen, "Coset Construction from Functional Integrals," Nucl. Phys. B 320 (1989) 625. 
[12] K. Gawedzki, "Classical origin of quantum group symmetries in WessZumino-Witten conformal field theory," Commun. Math. Phys. 139 (1991) 201.

[13] K. Gawedzki, "Conformal field theory: A case study," in Conformal field theory, Frontiers in Physics 102, eds. Nutku, Y., Saçlioglu, C., Turgut., Perseus Publishing, Cambridge Ma. 2000, pp. 1-55 [arXiv:hep-th/9904145.]

[14] K. Gawedzki, I. Todorov and P. Tran-Ngoc-Bich, "Canonical quantization of the boundary Wess-Zumino-Witten model," Commun. Math. Phys. 248 (2004) 217 arXiv:hep-th/0101170].

[15] K. Gawedzki, "Boundary WZW, G/H, G/G and CS theories," Annales Henri Poincare 3 (2002) 847 arXiv:hep-th/0108044].

[16] D. Karabali, Q. H. Park, H. J. Schnitzer and Z. Yang, "A GKO Construction Based on a Path Integral Formulation of Gauged Wess-Zumino-Witten Actions," Phys. Lett. B 216 (1989) 307.

[17] G. W. Moore and N. Seiberg, "Taming the Conformal Zoo," Phys. Lett. B 220 (1989) 422.

[18] G. Sarkissian, "Generalised permutation branes on a product of cosets $\mathrm{G}(\mathrm{k}(1)) / \mathrm{H} \quad \mathrm{x} \quad \mathrm{G}(\mathrm{k}(2)) / \mathrm{H}, " \quad$ Nucl. $\quad$ Phys. $\quad$ B $\mathbf{7 4 7}$ (2006) 423 arXiv:hep-th/0601061].

[19] G. Sarkissian, "Canonical quantization of the WZW model with defects and Chern-Simons theory," Int. J. Mod. Phys. A 25 (2010) 1367 arXiv:0907.3395 [hep-th]].

[20] G. Sarkissian, "Defects in G/H coset, G/G topological field theory and discrete Fourier-Mukai transform," Nucl. Phys. B 846 [PM] (2011) 338-357, arXiv:1006.5317 [hep-th].

[21] E. Witten, "Nonabelian bosonization in two dimensions," Commun. Math. Phys. 92 (1984) 455.

[22] E. Witten, "Quantum field theory and the Jones polynomial," Commun. Math. Phys. 121 (1989) 351. 